\title{
$C^{*}$-Algebras and Thermodynamic Formalism
}

\section{Rui Exel}

Departamento de Matemática, UFSC, Florianópolis, Brasil.

\section{Artur O. Lopes}

Instituto de Matemática, UFRGS, Porto Alegre, Brasil.

\begin{abstract}
We show a relation of the KMS state of a certain $C^{*}$ Algebra $\mathcal{U}$ with the Gibbs state of Thermodynamic Formalism. More precisely, we consider here the shift $T: X \rightarrow X$ acting on the Bernoulli space $X=\{1,2, \ldots, k\}^{\mathbf{N}}$ and $\mu$ a Gibbs state defined by a Holder continuous potential $p: X \rightarrow \mathbf{R}$, and $\mathcal{L}^{2}(\mu)$ the associated Hilbert space.

Consider the $C^{*}$-Algebra $\mathcal{U}=\mathcal{U}(\mu)$, which is a sub- $C^{*}$-Algebra of the $C^{*}$-Algebra of linear operators in $\mathcal{L}^{2}(\mu)$ which will be precisely defined later.

We call $\mu$ the reference measure.

Consider a fixed Holder potential $H>0$ and the $C^{*}$-dynamical system defined by the associated homomorphism $\sigma_{t}$. We are interested in describe for such system the KMS states $\phi_{\beta}$ for all $\beta \in \mathbf{R}$.

We show a relation of a new Gibbs probability $\nu_{\beta}$ to a KMS state $\phi_{\nu_{\beta}}=\phi_{\beta}$, in the $C^{*}$-Algebra $\mathcal{U}=\mathcal{U}(\mu)$, for every value $\beta \in \mathbf{R}$, where $\beta$ is the parameter that defines the time evolution associated to a homomorphism $\sigma_{t}=\sigma_{\beta i}$ defined by the potential $H$. We show that for each real $\beta$ the KMS state is unique.

The probability $\nu_{\beta}$ is the Gibbs state for the potential $-\beta \log H$.

The purpose of the present work is to explain (for an audience which is more oriented to Dynamical System Theory) part the content of a previous paper written by the authors.
\end{abstract}

\section{Introduction}

In this paper we show a relation of the KMS state of a certain $C^{*}$-Algebra $\mathcal{U}$ [4] [24] [9] with the Gibbs state of Thermodynamic Formalism [23] [2] [29]. The purpose of this work is to explain for an audience which is more oriented to Dynamical System Theory part the content of the paper [10]. See also $[25],[26]$ for related material. 
R. Bowen, D. Ruelle and Y. Sinai are the founders of what is called in our days Thermodynamic Formalism Theory (see [23] [29]).

We will present initially the precise definitions we are going to consider.

We point out that we show here only the uniqueness part of the results in [7]. The existence is based on the paper [30] which is of Functional Analysis nature.

We consider here an expanding transformation $T: X \rightarrow X$ (to simplify ideas one can consider the particular case where $T$ is the shift acting on the Bernoulli space $\left.X=\{1,2, \ldots, k\}^{\mathbf{N}}\right)$. Consider $\mu$ a Gibbs state defined by a Holder continuous potential $p: X \rightarrow \mathbf{R}$, and $\mathcal{L}^{2}(\mu)$ the associated Hilbert space.

Consider the $C^{*}$-Algebra $\mathcal{U}=\mathcal{U}(\mu)$, which is a sub- $C^{*}$-Algebra of the $C^{*}$-Algebra of linear operators in $\mathcal{L}^{2}(\mu)$ which will be precisely defined later.

We call $\mu$ the reference measure.

Consider a fixed Holder potential $H>0$ and the $C^{*}$-dynamical system defined by the associated $\sigma_{z}$. We are interested in describe for such system the KMS states $\phi_{\beta}$ for all $\beta \in \mathbf{R}$.

We show a relation of a new Gibbs probability $\nu_{\beta}$ to a KMS state $\phi_{\nu_{\beta}}=$ $\phi_{\beta}$, in the $C^{*}$-Algebra $\mathcal{U}=\mathcal{U}(\mu)$, for every value $\beta \in \mathbf{R}$, where $\beta$ is the parameter that defines the time evolution associated to a homorphism $\sigma_{t}=$ $\sigma_{\beta i}$ defined by the potential $H$. We show that for each real $\beta$ the KMS state is unique in Theorem 2.2.

The probability $\nu_{\beta}$ is the Gibbs state for the potential $-\beta \log H$.

Given a potential $H$, we say the potential $\tilde{H}$ is cohomologous to $H$, if there is $V$ such that $\log \tilde{H}=\log H-V+V \circ T$.

After we present our main results for Holder potentials in section 2.1, in section 2.2 we consider a non-Holder potential $H$ and we will make an analysis of phase transition nature (which do not occur at $C^{*}$ Algebra level, in this case) associated to the KMS problem in a case where $H$ can attain the value 1 (and where there is phase transition at Thermodynamic Formalism level).

\section{KMS and Gibbs states}

We denote $C(X)$ the space of continuous functions on $X$ taking values on the complex numbers where $(X, d)$ is a compact metric space. 
Consider the Borel sigma-algebra $\mathcal{B}$ over $X$ and a continuous transformation $T: X \rightarrow X$. Denote by $\mathcal{M}(T)$ the set of invariant probabilities for $T$. We assume that $T$ is an expanding map.

We refer the reader to [2] [27] [28] [29] [21] for general definitions and properties of Thermodynamic Formalism and expanding maps.

Possible examples of such transformations (for which that are a lot of nice results [28]) are the shift in the Bernoully space and also $C^{1+\alpha_{-}}$ transformations of the circle such that $\left|T^{\prime}(x)\right|>c>1$, where || is the usual norm (one can associate the circle to the interval $[0,1$ ) in a standard way) and $c$ is a constant.

The geodesic flow in compact constant negative curvature surfaces induces in the boundary of Poincaré disk a Markov transformation $G$ such that for some $n$, we have $G^{n}=T$, and where $T$ is continuous expanding and acts on the circle (see [3]). Our results can be applied for such $T$.

We denote by $\mathcal{H}=\mathcal{H}_{\alpha}$ the set of $\alpha$-Holder functions taking complex values, where $\alpha$ is fixed $0<\alpha \leq 1$.

For each $\nu \in \mathcal{M}(T), h(\nu)$ denotes the the Shanon-Kolmogorov entropy of $\nu$ and $h(T)=\sup \{h(\nu) \mid \nu \in \mathcal{M}(T)\} . h(T)$ is called the topological entropy of $T$.

We denote by $\mu$ a fixed Gibbs state for a real Holder potential $\log p$ : $X \rightarrow \mathbf{R}$. We suppose $\log p$ is already normalized [2][29], in the sense that, if $\mathcal{L}_{p}$ denotes the Ruelle-Perron-Frobenius operator for $\log p$, that is for any $f: X \rightarrow \mathbf{C}$, and all $x \in X$, we have $\left(\mathcal{L}_{p}(f)\right)(x)=\sum_{T(z)=x} p(z) f(z)$, then we assume that $\mathcal{L}_{p}(1)(x)=\sum_{T(z)=x} p(z)=1$ and $\mathcal{L}_{p}^{*}(\mu)=\mu$.

We will show later that the index $\Lambda(x)=p(x)^{-1}$ for the $C^{*}$-algebra associated to $\mu$.

As an interesting example we mention the case where $T$ has degree $k$, that is, for each $x \in X$ there exists exactly $k$ different solutions $z$ for $T(z)=x$. We call each such $z$ a pre-image of $x$.

If $T$ has degree $k$ and in the particular case where $\mu$ is the maximal entropy measure (that is, $h(\mu)=h(T)=\log k$ ), then $p=1 / k$.

We do not have to assume that the number of preimages of each $x$ is constant. In order to simplify the arguments in our proofs we will assume from now on that $T$ has degree $k$. The reader can easily realize the changes that have to be done in order to consider the general case.

One can consider alternatively in Thermodynamic Formalism $\mathcal{L}_{p}$ acting on $C(X)$ or on $\mathcal{H}_{\alpha}$. Different spectral properties for $\mathcal{L}_{p}$ occur in each one of these two cases (see[2][28]). 
We will consider in the sequel a fixed real Holder-continuous positive potential $H: X \rightarrow \mathbf{R}$ and $\mathcal{L}_{H, \beta}, \beta \in \mathbf{R}$ the Ruelle-Perron-Frobenius operator for $-\beta \log H$, that is, for each continuous $f$ we have by definition

$$
\mathcal{L}_{H, \beta}(f)(x)=\sum_{T(z)=x} H(z)^{-\beta} f(z) .
$$

We denote by $\lambda_{H, \beta} \in \mathbf{R}$ the largest eigenvalue of $\mathcal{L}_{H, \beta}$. We also denote $\nu_{H, \beta}$ the unique probability such that $\mathcal{L}_{H, \beta}^{*}\left(\nu_{H, \beta}\right)=\lambda_{H, \beta} \nu_{H, \beta}$, and $h_{H, \beta}$ the unique function $h \in C(X)$ such that $\int h d \nu_{H, \beta}=1$ and $\mathcal{L}_{H, \beta}(h)=\lambda_{H, \beta} h$.

$h_{H, \beta}$ is a real positive Holder function.

The hypothesis about $H$ and $p$ being Holder in the Statistical Mechanics setting means that in the Bernoulli space the interactions between spins in neighborhoods positions decrease very fast [19] [20]. In section 2.3 we will consider a non-Holder potential $H$ where in this case it will appear a phase-transition phenomena. This model is known as the Fisher-Felderhof model [12], [19], [20], [11]. In this case the interactions do not decrease so fast. We return now to the Holder case.

It is well known the variational principle for such potential $-\beta \log H$,

$$
P_{H}(\beta)=\log \lambda_{H, \beta}=\sup \left\{h(\nu)+\int(-\beta \log H) d \nu \mid \nu \in \mathcal{M}(T)\right\} .
$$

The probability $\mu_{H, \beta}=h_{H, \beta} \nu_{H, \beta} \in \mathcal{M}(T)$ and satisfies

$$
\begin{gathered}
\sup \left\{h(\nu)+\int(-\beta \log H) d \nu \mid \nu \in \mathcal{M}(T)\right\}= \\
h\left(\mu_{H, \beta}\right)+\int(-\beta \log H) d \mu_{H, \beta} .
\end{gathered}
$$

Definition 2.1: The probability $\mu_{\beta}=h_{H, \beta} \nu_{H, \beta}$ is called equilibrium state for the function $-\beta \log H$ where $\beta$ and $H$ are fixed.

Definition 2.2: The probability $\nu_{H, \beta}$ is called eigenmeasure or Gibbs state for the function $-\beta \log H$ where $\beta$ and $H$ are fixed. It satisfies

$$
\mathcal{L}_{H, \beta}^{*}\left(\nu_{H, \beta}\right)=\lambda_{H, \beta} \nu_{H, \beta} \text {. }
$$

The probability $\mu_{H, \beta}$ is unique for the variational problem and $\nu_{H, \beta}$ is unique for the the eigenmeasure problem associated to the value $\lambda_{H, \beta}$, if $p$ and $H$ are Holder. If we do not assume $p$ and $H$ Holder then there exist counterexamples for uniqueness in both cases [19] [20]. We will return to this point later.

For some reason the eigen-probabilities have a distinguished role here, but not the equilibrium states. 
$P_{H}(\beta)$ is called the pressure of $-\beta \log H$ (or sometimes Free-Energy) and is a convex analytic function of $\beta$. If $H>1$ then $\lim _{\beta \rightarrow \infty} P_{H}(\beta)=-\infty$ and $\lim _{\beta \rightarrow-\infty} P_{H}(\beta)=\infty$.

Under the hypothesis $H>0$ sometimes there is no real $\beta$ such that $P_{H}(\beta)=0$.

If $T$ has degree $k$ and in the particular case where $\mu$ is the maximal entropy measure (that is, $h(\mu)=h(T)=\log k$ ), then $p=1 / k$.

Definition 2.3: Denote by $S: \mathcal{L}^{2}(\mu) \rightarrow \mathcal{L}^{2}(\mu)$ the Koopman operator where for $\eta \in \mathcal{L}^{2}(\mu)$ we define $(S \eta)(x)=\eta(T(x))$. such $S$ defines a linear operator in $\mathcal{L}^{2}(\mu)$.

In Thermodynamic Formalism it is usual to consider the Koopman operator acting on $\mathcal{L}^{2}(\mu)$ (the space of complex square integrable functions over $\mathcal{L}^{2}(\mu)$ ), and it is well known that its adjoint (over $\mathcal{L}^{2}(\mu)$ ) is the operator $\mathcal{L}_{p}$ acting on $\mathcal{L}^{2}(\mu)$. As we assume $X$ is compact, any continuous function $f$ is in $\mathcal{L}^{2}(\mu)$.

We denote by $L\left(\mathcal{L}^{2}(\mu)\right)$, the set of linear operators over the linear space $\mathcal{L}^{2}(\mu)$; an example: $S \in L\left(\mathcal{L}^{2}(\mu)\right)$.

Definition 2.4: Another important class of linear operators is $M_{f}$ : $\mathcal{L}^{2}(\mu) \rightarrow \mathcal{L}^{2}(\mu)$, for a given fixed $f \in C(X)$, and defined by $M_{f}(\eta)(x)=$ $f(x) \eta(x)$, for any $\eta$ in $\mathcal{L}^{2}(\mu)$.

In order to simplify the notation, sometimes we denote by $f$ the linear operator $M_{f}$.

Note that for $M_{f}$ and $M_{g}, f, g \in C(X)$, the product operation satisfies $M_{f} \circ M_{g}=M_{f . g}$, where . means multiplication over the complex field $\mathbf{C}$.

Note that the $*$ operation applied on $M_{f}, f \in C(X)$, is given by $M_{f}^{*}=$ $M_{\bar{f}}$, where $\bar{z}$ is the complex conjugated of $z \in \mathbf{C}$. In this sense, $M_{f}^{*}$ is the adjoint operator of $M_{f}$ over $\mathcal{L}^{2}(\mu)$.

The main point for our choice of $\mu$ as eigen-probability for $\mathcal{L}_{p}^{*}$, is that in $\mathcal{L}^{2}(\mu)$, the dual of the Koopman operator $S$ is the operator $\mathcal{L}_{p}=S^{*}$ acting on $\mathcal{L}^{2}(\mu)$. Indeed, for any $f, g$ we have

$$
\begin{aligned}
& \int f(g \circ T) d \mu=\int f(g \circ T) d \mathcal{L}_{p}^{*}(\mu) \\
& =\int \mathcal{L}_{p}(f(g \circ T)) d \mu=\int \mathcal{L}_{p}(f) g d \mu .
\end{aligned}
$$

$L\left(\mathcal{L}^{2}(\mu)\right)$, the set of linear operators over $\mathcal{L}^{2}(\mu)$, is a very important $C^{*}$-Algebra. We will analyze here a sub- $C^{*}$-Algebra of such $C^{*}$-Algebra 
(defined with the above operations . and $*$ ), more precisely the $C^{*}$-Algebra $\mathcal{U}$.

Definition 2.5: We denote by $\alpha: C(X) \rightarrow C(X)$ the linear operator such that for any $f$, we have $\alpha(f)=f \circ T$.

We have to show how the operators $S$ and $M_{f}$ acting on $\mathcal{L}^{2}(\mu)$ interact with the operators $\mathcal{L}_{p}$ and $\alpha$ acting on $C(X)$.

One can easily see that $\alpha\left(M_{f}\right)=M_{f \circ \sigma}$. This is the first relation.

In the simplified notation (we identify $M_{f}$ with $f$ ), one can read last expression as $\alpha(f)=f \circ T$.

Definition 2.6: Consider the $C^{*}$-Algebra contained in $L\left(\mathcal{L}^{2}(\mu)\right)$ and generated by the elements of the form $M_{f} S^{n}\left(S^{*}\right)^{n} M_{q}$, where $n \in \mathbf{N}$ and $f, g \in C(X)$. We denote such $C^{*}$-Algebra by $\mathcal{U}=\mathcal{U}(\mu, T)$. We call $\mathcal{U}$ the $C^{*}$-Algebra associated to $\mu$.

Each element $a$ in $\mathcal{U}$ is the limit of finite sums $\sum_{i} M_{f_{i}} S^{n_{i}}\left(S^{*}\right)^{n_{i}} M_{g_{i}}$.

Note that $f \rightarrow M_{f}$ defines a linear injective function of $C(X)$ on $\mathcal{U}$.

We have basic relations in such $C^{*}$-Algebra $\mathcal{U}$ :

a) $\left(S^{*}\right)^{n} S^{n}=1$, for all $n \in \mathbf{N}$ (it follows from $S^{*} S=1$ ).

proof: for any $\eta \in \mathcal{L}^{2}(\mu)$, we have

$$
\begin{aligned}
& S^{*} S(\eta)(x)=\mathcal{L}_{p}\left(\eta(T(.))(x)=\sum_{T(y)=x} p(y) \eta(T(y))\right. \\
& =\sum_{T(y)=x} p(y) \eta(x)=\eta(x) .
\end{aligned}
$$

b) $\left(S^{*}\right)^{n} M_{f} S^{n}=M_{\mathcal{L}_{p}^{n}(f)}$, for all $n \in \mathbf{N}, f \in C(X)$ (it follows from $\left.S^{*} M_{f} S=M_{\mathcal{L}_{p}(f)}\right)$.

proof: for any $\eta \in \mathcal{L}^{2}(\mu)$, we have

$$
S^{*} M_{f} S(\eta)(x)=\mathcal{L}_{p}(f \eta(T(.)))(x)=\mathcal{L}_{p}(f)(x) \eta(x) .
$$

and finally

c) $S M_{f}=\alpha(f) S$ for any continuous $f$, that is, for any $\eta \in \mathcal{L}^{2}(\mu)$, $S M_{f}(\eta)=f \circ T . \eta \circ T=\alpha(f) . S(\eta)$.

Remark: If we consider the $C^{*}$-algebra generated $M_{f} S^{m}\left(S^{*}\right)^{n} M_{g}$, where $n, m \in \mathbf{N}$ and $f, g \in C(X)$, we have a different setting (which is usually called a Vershik $C^{*}$-algebra) which was consider in another paper by R. Exel [7]. In this case, the KMS state exists only for one value of $\beta$. 
We now return to our setting.

An extremely important result will be shown in Lemma 2.1 which claims that there exists functions $u_{i}, i \in\{1,2, . ., k\}$, such that

$$
\sum_{i=1}^{k} M_{u_{i}} S S^{*} M_{u_{i}}=1 \text {. }
$$

We denote by $\operatorname{Aut}(\mathcal{U})$ the set of automorphism of the $C^{*}$-Algebra $\mathcal{U}$.

Definition 2.7: Given a positive function $H$ we define the group homomorphism $\sigma_{t}$, where for each $t \in \mathbf{R}$ we have $\sigma_{t} \in \operatorname{Aut}(\mathcal{U})[\mathrm{BP}][\mathrm{P}]$, is defined by:

a) for each fixed $t \in \mathbf{R}$ and any $M_{f}$, we have $\sigma_{t}\left(M_{f}\right)=M_{f}$,

b) for each fixed $t \in \mathbf{R}$, we have $\sigma_{t}(S)=M_{H^{i t}} \circ S$, , in the sense that $\left(\sigma_{t}(S)(\eta)\right)(x)=H^{i t}(x) \eta(T(x)) \in \mathcal{L}^{2}(\mu)$, for any $\eta \in \mathcal{L}^{2}(\mu)$.

The value $t$ above is related to temperature and not time, more precisely we are going to consider bellow $t=\beta i$ where $\beta$ is related to the inverse of temperature in Thermodynamic Formalism (or Statistical Mechanics).

It can be shown that for each $t$ fixed, we just have to define $\sigma_{t}$ over the generators of $\mathcal{U}$ in order to define $\sigma_{t}$ uniquely on $\mathcal{U}$. In this way a) and b) above define $\sigma_{t}$.

We will assume in this section from now on that $H$ is Holder in order we can use the strong results of Thermodynamic Formalism.

Remark 1: Note that for $\eta \in \mathcal{L}^{2}(\mu)$, we have

$$
\begin{gathered}
\left(\sigma_{t}\left(S^{2}\right) \eta\right)(x)=\sigma_{t}\left(M_{H^{i t}}(\eta \circ T)\right)(x)= \\
M_{H^{i t}} M_{H^{i t} \circ T}\left(\eta \circ T^{2}\right)(x),
\end{gathered}
$$

therefore $\sigma_{t}\left(S^{2}\right)=H^{t i}(H \circ T)^{t i} S^{2}$. It follows easily by induction that

$$
\sigma_{t}\left(S^{n}\right)=\Pi_{j=0}^{n-1}\left(H \circ T^{j}\right)^{t i} S^{n} .
$$

Taking dual in both sides of the above expression we get other important relation

$$
\sigma_{t}\left(\left(S^{*}\right)^{n}\right)=\left(S^{*}\right)^{n} \Pi_{j=0}^{n-1}\left(H \circ T^{j}\right)^{-t i} .
$$

In terms of the formalism of $C^{*}$-dynamical systems, the positive function $H$ defines the dynamics of the evolution with time $t \in \mathbf{R}$ of a $C^{*}$-dynamical system. Our purpose is to analyze such system for each pair $(H, \beta)$.

Definition 2.8: An element $a$ in a $C^{*}$-Algebra is positive, if it is of the form $a=b b^{*}$ with $b$ in the $C^{*}$-Algebra. 
Definition 2.9: By definition a " $C^{*}$-dynamical system state" is a linear functional $\phi: \mathcal{U} \rightarrow \mathbf{C}$ such that

a) $\phi\left(M_{1}\right)=1$

b) $\phi(a)$ is a positive real number for each positive element $a$ on the $C^{*}$-Algebra $\mathcal{U}$.

A " $C^{*}$-dynamical system state" $\phi$ in $C^{*}$-dynamical systems plays the role of a probability $\nu$ in Thermodynamic Formalism. For a fixed $H$, we have a dynamic temporal evolution defined by $\sigma_{t}$ where $t \in \mathbf{R}$.

Definition 2.10: An element $a \in \mathcal{U}$ is called analytic for $\sigma$ if $\sigma_{t}(a)$ has an analytic extension from $t \in \mathbf{R}$ to all $t \in \mathbf{C}$.

Definition 2.11: For a fixed $\beta \in \mathbf{R}$ and $H$, by definition, $\phi$ is a KMS state associated to $H$ and $\beta$ in the $C^{*}$-Algebra $\mathcal{U}(\mu, T)$, if $\phi$ is a $C^{*}$ dynamical system state, such that for any $b \in \mathcal{U}$ and any analytic $a \in \mathcal{U}$ we have

$$
\phi(a . b)=\phi\left(b . \sigma_{\beta i}(a)\right) .
$$

For $H$ and $\beta$ fixed, we denote a KMS state by $\phi_{H, \beta}$ and we leave $\phi$ for a general $C^{*}$-dynamical system state.

It is easy to see that for $H$ and $\beta$ fixed, the condition

$$
\phi(a . b)=\phi\left(b . \sigma_{\beta i}(a)\right)
$$

is equivalent to $\forall \tau \in \mathbf{C}$,

$$
\phi\left(\sigma_{\tau}(a) . b\right)=\phi\left(b . \sigma_{\tau+\beta i}(a)\right) .
$$

It follows from section 8.12 in [24] that if $\phi$ is a KMS state for $H, \beta$, then for any analytic $a \in \mathcal{U}$, we have that $\tau \rightarrow \phi\left(\sigma_{\tau}(a)\right)$ is a bounded entire function and therefore constant. In this sense $\phi$ is stationary.

Note that the KMS state, in principle, could depend of the initially chosen $\mu$ because we are considering $\mathcal{L}^{2}(\mu)$ when defining $\mathcal{U}$, but in the end it will be defined by a measure that depends only in $\beta$ and $H$.

We point out that it can be shown that in order to characterize $\phi$ as a KMS state we just have to check the condition $\phi(a . b)=\phi\left(b . \sigma_{\beta i}(a)\right)$ for $a, b$ the linear generators of $\mathcal{U}$, that is, $a$ of the form $M_{f_{1}} S^{n}\left(S^{*}\right)^{n} M_{g_{1}}$ and $b$ of the form $M_{f_{2}} S^{m}\left(S^{*}\right)^{m} M_{g_{2}}$.

A natural question is: for a given $\beta$ and $H$, when the KMS state $\phi_{H, \beta}$ exist and when it is unique?

We are interested mainly in uniqueness. We will explain this point more carefully later. The case of temperature zero (or, $\beta=\infty$ ) is consider in $[10]$. 
Remark 2: Note that when $\phi=\phi_{H, \beta}$ is a KMS state, $\phi(a . g)=$ $\phi\left(a . \sigma_{\beta i}(g)\right)=\phi(g . a)$, for any $g \in C(X)$ and $a \in \mathcal{U}$.

Our purpose here is to show how to associate in a unique way each KMS state $\phi_{H, \beta}$ to the eigenmeasure $\nu_{H, \beta}$ defined before.

Remeber that over $\mathcal{L}^{2}(\mu)$ the operator $\mathcal{L}_{p}$ is adjoint of the operator $f \rightarrow$ $f \circ T$.

We call $\Lambda(x)=p(x)^{-1}$ the index and we denote by

$$
\Lambda^{[n]}(x)=\left(p(x) p(T(x)) \ldots p\left(T^{n-1}(x)\right)\right)^{-1} .
$$

Now we define $E: \mathcal{U} \rightarrow C(X)$, a conditional expectation (that is $E \circ E=$ $E)$, such that

a) $E\left(M_{f}\right)=M_{f}$,

b) $E(a)$ of any positive element $a$ is a positive number in $\mathbf{R}$ by:

$$
E\left(M_{f} S^{n}\left(S^{*}\right)^{n} M_{g}\right)=\frac{1}{\Lambda^{[n]}} M_{f g} .
$$

This expression defines $E$ (taking limits) over all $\mathcal{U}$.

Our main theorem says:

Theorem 2.1: If $H$ is Holder positive and $\mu$ is a Gibbs state for $p$ Holder, then for any given $\beta \in \mathbf{R}$, a KMS state in $\mathcal{U}(\mu) \phi$ exists, it is unique and of the form

$$
\phi_{H, \beta}(a)=\int E(a) d \nu_{H, \beta}, \quad \forall a \in \mathcal{U},
$$

where $\nu_{H, \beta}$ is the eigenmeasure for $\mathcal{L}_{H, \beta}^{*}$.

Proof of Theorem 2.1:

The existence of the KMS follows immediately from taking $e_{n}=S^{n} S^{n *}$ and $E_{n}=\alpha^{n} \mathcal{L}_{p}^{n}$ in section 1 . We want in this section to show precisely how one can associate a Gibbs measure to such $\phi_{H, \beta}$.

First we outline the main results of the previous section but now in the particular notation that it is used in the context of Thermodynamic Formalism. We believe that with this procedure we will help the understanding for the reader familiar with Thermodynamic Formalism but not so much with $C^{*}$-Algebra Theory. Alternatively, the reader can go directly to Theorem 2.5 to find the proof of the main result of this section: uniqueness of the KMS state for the $C^{*}$-Algebra $\mathcal{U}$.

In order to simplify the notation, for $H$ and $\beta$ fixed we denote $\nu_{H, \beta}$ by $\nu_{\beta}, \mathcal{L}_{H, \beta}$ by $\mathcal{L}_{\beta}, \lambda_{H, \beta}$ by $\lambda_{\beta}$ and $h_{H, \beta}$ by $h_{\beta}$. 
We will denote $\phi_{H, \beta}$, the KMS state for $H, \beta$, by $\phi_{\beta}$.

We will leave the terminology $\phi_{\beta}$ for the one associated to the Gibbs state $\nu_{\beta}$ and use $\phi$ for the one associated to the above defined $\nu$

Suppose $\phi$ is a KMS state, where $H, \beta$ are fixed.

First we will show that if $\phi$ is a KMS state for $H$ and $\beta$, then there exist a measure $\nu$ such that

a) for all $n \in \mathbf{N}$

$$
\phi\left(M_{f} S^{n}\left(S^{*}\right)^{n}\right)=\int \frac{f}{\Lambda^{[n]}} d \nu,
$$

and

b) for all $f_{1}, f_{2}, g_{1}, g_{2} \in C(X)$ and all $n, m \in \mathbf{N}$.

$$
\begin{gathered}
\phi\left(M_{f_{1}} S^{n}\left(S^{*}\right)^{n} M_{g_{1}} \cdot \sigma_{\beta i}\left(M_{f_{2}} S^{m}\left(S^{*}\right)^{m} M_{g_{2}}\right)=\right. \\
\phi\left(M_{f_{2}} S^{m}\left(S^{*}\right)^{m} M_{g_{2}} \cdot M_{f_{1}} S^{n}\left(S^{*}\right)^{n} M_{g_{1}}\right) .
\end{gathered}
$$

Given the KMS state $\phi$, then $\phi\left(M_{f}\right)=\phi(f)$ defines a continuous positive linear functional over $C(X)$ such that $\phi\left(M_{1}\right)=1$. Therefore by Riesz Theorem, there exists a probability $\nu$ such that for any $f \in C(X)$ we have $\phi(f)=\int f d \nu=\int f S^{0}\left(S^{*}\right)^{0} d \nu$.

This $\nu$ is our candidate to be the one associated to $\phi$. The above definition takes in account just $n=0$ in a) above. Remains the question: what conditions we should impose on $\nu$ (defined from $\phi$ as above) in order to make $\phi$ a KMS state for $H, \beta$ ? That is, when $\nu$ satisfies a) and b) above?

Now we will show a recurrence relation associated to any KMS state $\phi$ for $H, \beta$ :

Lemma 2.1: $\phi\left(f S^{n}\left(S^{*}\right)^{n}\right)=\int f \Lambda^{-[n]} d \nu$, for any $f \in C(X)$,

\section{Proof:}

The lemma will follow from $\phi\left(f S^{n}\left(S^{*}\right)^{n}\right)=\phi\left(\left(\Lambda \circ T^{n}\right) f S^{n+1}\left(S^{*}\right)^{n+1}\right)$.

We need a preliminary estimate before proving the lemma.

For the transformation $T$, consider a partition $A_{1}, \ldots, A_{k}$ of $X$ such that $T$ is injective in each $A_{i}$. Our proof bellow is for the shift in the Bernouilli space. In the case of the Bernoulli space with $k$ symbols $A_{i}$ is the cylinder $\bar{i}$ with first coordinate $i$. Now we consider a partition of unity given by $k$ non-negative functions $v_{1}, \ldots, v_{k}$ such that each $v_{i}(x)=I_{\bar{i}}(x)$ (the indicator function of the cylinder $\bar{i}$ ) which has support on $A_{i}$ and $\sum_{i=1}^{k} v_{i}(x)=1$ for all $x \in X$. In the case $X$ is the unitary circle and $T$ is expansive, using a conjugacy with the shift, we obtain similar results. 
Denote now the functions $u_{i}$ given by

$$
u_{i}(x)=\left(v_{i}(x) \Lambda(x)\right)^{1 / 2}=\left(v_{i}(x) p(x)^{-1}\right)^{1 / 2},
$$

for each $i \in\{1, \ldots, k\}$, so, $\sum_{i=1}^{k} u_{i}^{2}(x)=\Lambda(x)=p(x)^{-1}$, for any $x \in X$.

An easy computation shows that $\sum_{i=1}^{k} M_{u_{i}} S S^{*} M_{u_{i}}=1$. Indeed, if $x$ is in the cylinder $\bar{i}$, then given $\eta$, we have

$$
\left[M_{u_{i}} S S^{*} M_{u_{i}}(\eta)\right](x)=u_{i}(x) \sum_{\{z \mid \sigma(z)=\sigma(x)\}} p(z) u_{i}(z) \eta(z)=\eta(x) .
$$

Now

$$
\begin{gathered}
S^{n}\left(S^{*}\right)^{n}=S^{n} 1\left(S^{*}\right)^{n}=S^{n}\left[\sum_{i=1}^{k}\left(M_{u_{i}} S S^{*} M_{u_{i}}\right)\right]\left(S^{*}\right)^{n}= \\
\sum_{i=1}^{k}\left(S^{n}\left(M_{u_{i}} S S^{*} M_{u_{i}}\right)\left(S^{*}\right)^{n}\right) .
\end{gathered}
$$

Now we use the relations $S^{n} M_{f}=M_{\alpha^{n}(f)} S^{n}$ and $M_{f}\left(S^{*}\right)^{n}=\left(S^{*}\right)^{n} M_{\alpha^{n}(f)}$ in last expression and we get

$$
\begin{aligned}
S^{n}\left(S^{*}\right)^{n} & =\sum_{i=1}^{k} M_{\alpha^{n}\left(u_{i}\right)} S^{n} S S^{*}\left(S^{*}\right)^{n} M_{\alpha^{n}\left(u_{i}\right)} \\
& =\sum_{i=1}^{k} M_{\alpha^{n}\left(u_{i}\right)} S^{n+1}\left(S^{*}\right)^{n+1} M_{\alpha^{n}\left(u_{i}\right)}
\end{aligned}
$$

Now we will prove the lemma.

Using last expression and then Remark 2 for $g=\alpha^{n}\left(u_{i}\right) \in C(X)$ and $a=S^{n+1}\left(S^{*}\right)^{n+1}$ we get

$$
\begin{gathered}
\phi\left(M_{f} S^{n}\left(S^{*}\right)^{n}\right)=\phi\left(M_{f} \sum_{i=1}^{k} M_{\alpha^{n}\left(u_{i}\right)} S^{n+1}\left(S^{*}\right)^{n+1} M_{\alpha^{n}\left(u_{i}\right)}\right)= \\
\phi\left(M_{f} \sum_{i=1}^{k} M_{\alpha^{n}\left(u_{i}\right)} M_{\alpha^{n}\left(u_{i}\right)} S^{n+1}\left(S^{*}\right)^{n+1}\right)= \\
\phi\left(M_{f} \sum_{i=1}^{k} M_{\alpha^{n}\left(u_{i}\right)^{2}} S^{n+1}\left(S^{*}\right)^{n+1}\right)=
\end{gathered}
$$




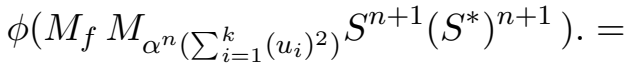

$$
\begin{aligned}
& \phi\left(M_{f}\left(\Lambda \circ T^{n}\right) S^{n+1}\left(S^{*}\right)^{n+1}\right)
\end{aligned}
$$

This shows the claim of the lemma.

We denote by $E_{n}$ the operator $\alpha^{n} \mathcal{L}_{p}^{n}$ acting on $C(X)$. This operator $E_{n}$ acting on $C(X)$ is a conditional expectation $E_{n}(f)=\mathcal{E}\left(f, \mathcal{R}_{n}\right)$, where $\mathcal{R}_{n}=\left\{f \circ T^{n} \mid f \in C(X)\right\}$. It is well known that $E_{n}: C(X) \rightarrow \mathcal{R}_{n}$, and $E_{n}(f g)=f E_{n}(g)$, if $f \in \mathcal{R}_{n}$.

It is known that if $n>m$, then $E_{n} \circ E_{m}=E_{n}$.

Note that for any $n$, we have $E_{n}(1)=1$.

Now we will introduce the restriction on $\nu$ defined by the time evolution of $\sigma_{t}$, that is condition b) above.

Lemma 2.2: The KMS condition

$$
\begin{gathered}
\phi\left(M_{f_{1}} S^{n}\left(S^{*}\right)^{n} M_{g_{1}} \cdot \sigma_{\beta i}\left(M_{f_{2}} S^{m}\left(S^{*}\right)^{m} M_{g_{2}}\right)=\right. \\
\phi\left(M_{f_{2}} S^{m}\left(S^{*}\right)^{m} M_{g_{2}} \cdot M_{f_{1}} S^{n}\left(S^{*}\right)^{n} M_{g_{1}}\right) .
\end{gathered}
$$

for any $n, m \in \mathbf{N}, f_{1}, f_{2}, g_{1}, g_{2} \in C(X)$, is equivalent to the condition

$$
\int f_{2} g_{1} E_{m}\left(g_{2} f_{1}\right) d \nu=\int g_{2} f_{1} H^{\beta[m]} \Lambda^{-[n]} E_{m}\left(f_{2} g_{1} H^{-\beta[m]} \Lambda^{[n]}\right) d \nu,
$$

for any $m \in \mathbf{N}, f_{1}, f_{2}, g_{1}, g_{2} \in C(X)$.

\section{Proof:}

Suppose, first that $n \geq m$ (we consider the other case later).

From Remark 1 we have

$$
\sigma_{\beta i}\left(M_{f_{2}} S^{m}\left(S^{*}\right)^{m} M_{g_{2}}\right)=M_{f_{2}} H^{-\beta[m]} S^{m}\left(S^{*}\right)^{m} H^{\beta[m]} M_{g_{2}},
$$

where by definition $H^{\beta[m]}(x)=\Pi_{i=0}^{m-1} H\left(T^{i}(x)\right)^{\beta}$.

Therefore, the KMS condition imposed by $\sigma$ can be written as

$$
\begin{gathered}
\phi\left(M_{f_{2}} S^{m}\left(S^{*}\right)^{m} M_{g_{2}} \cdot M_{f_{1}} S^{n}\left(S^{*}\right)^{n} M_{g_{1}}\right)= \\
\phi\left(M_{f_{1}} S^{n}\left(S^{*}\right)^{n} M_{g_{1}} \cdot M_{f_{2}} H^{-\beta[m]} S^{m}\left(S^{*}\right)^{m} H^{\beta[m]} M_{g_{2}}\right) .(* * * * *)
\end{gathered}
$$

As we assume $n \geq m$, considering the right hand-side of the expression above we get

$$
\begin{gathered}
\phi\left(M_{f_{1}} S^{n}\left(S^{*}\right)^{n} M_{g_{1}} \cdot M_{f_{2}} H^{-\beta[m]} S^{m}\left(S^{*}\right)^{m} H^{\beta[m]} M_{g_{2}}\right)= \\
\phi\left(M_{f_{1}} S^{n}\left(S^{*}\right)^{n-m}\left(S^{*}\right)^{m} M_{g_{1}} \cdot M_{f_{2}} H^{-\beta[m]} S^{m}\left(S^{*}\right)^{m} H^{\beta[m]} M_{g_{2}}\right)=
\end{gathered}
$$




$$
\phi\left(M_{f_{1}} S^{n}\left(S^{*}\right)^{n-m} \mathcal{L}_{p}^{m}\left(g_{1} f_{2} H^{-\beta[m]}\right)\left(S^{*}\right)^{m} H^{\beta[m]} M_{g_{2}}\right) .
$$

As, for any continuous $g$, we have $g\left(S^{*}\right)^{m}=\left(S^{*}\right)^{m} \alpha^{m}(g)$, then take $g=\mathcal{L}_{p}^{m}\left(g_{1} f_{2} H^{-\beta[m]}\right) \in C(X)$, and using also lemma 1

$$
\begin{gathered}
\phi\left(M_{f_{1}} S^{n}\left(S^{*}\right)^{n-m} \mathcal{L}_{p}^{m}\left(g_{1} f_{2} H^{-\beta[m]}\right)\left(S^{*}\right)^{m} H^{\beta[m]} M_{g_{2}}\right)= \\
\phi\left(M_{f_{1}} S^{n}\left(S^{*}\right)^{n-m}\left(S^{*}\right)^{m} \alpha^{m} \mathcal{L}_{p}^{m}\left(g_{1} f_{2} H^{-\beta[m]}\right) H^{\beta[m]} M_{g_{2}}\right)= \\
\phi\left(M_{f_{1}} S^{n}\left(S^{*}\right)^{n} \alpha^{m} \mathcal{L}_{p}^{m}\left(g_{1} f_{2} H^{-\beta[m]}\right) H^{\beta[m]} M_{g_{2}}\right)= \\
\int f_{1} g_{2} H^{\beta[m]} E_{m}\left(g_{1} f_{2} H^{-\beta[m]}\right) \Lambda^{-[n]} d \nu
\end{gathered}
$$

Now, from the basic relation $\alpha^{m}(g) S^{m}=S^{m} g$, for $g=\mathcal{L}_{p}^{m}\left(g_{2} f_{1}\right) \in C(X)$ and Lemma 1, the left hand side of expression (*****) satisfies satisfies

$$
\begin{gathered}
\phi\left(M_{f_{2}} S^{m}\left(S^{*}\right)^{m} M_{g_{2}} \cdot M_{f_{1}} S^{n}\left(S^{*}\right)^{n} M_{g_{1}}\right)= \\
\phi\left(M_{f_{2}} S^{m}\left(S^{*}\right)^{m} M_{g_{2}} \cdot M_{f_{1}} S^{m} S^{n-m}\left(S^{*}\right)^{n} M_{g_{1}}\right)= \\
\phi\left(M_{f_{2}} S^{m} \mathcal{L}_{p}^{m}\left(g_{2} f_{1}\right) S^{n-m}\left(S^{*}\right)^{n} M_{g_{1}}\right)= \\
=\phi\left(M_{f_{2}} \alpha^{m} \mathcal{L}_{p}^{m}\left(g_{2} f_{1}\right) S^{m} S^{n-m}\left(S^{*}\right)^{n} M_{g_{1}}\right)= \\
=\phi\left(M_{f_{2}} \alpha^{m} \mathcal{L}_{p}^{m}\left(g_{2} f_{1}\right) S^{n}\left(S^{*}\right)^{n} M_{g_{1}}\right)= \\
\int f_{2} g_{1} E_{m}\left(g_{2} f_{1}\right) \Lambda^{-[n]} d \nu .
\end{gathered}
$$

From $(* * * * *)$ we finally get the expression

$$
\int f_{2} g_{1} E_{m}\left(g_{2} f_{1}\right) \Lambda^{-[n]} d \nu=\int g_{2} f_{1} H^{\beta[m]} E_{m}\left(f_{2} g_{1} H^{-\beta[m]}\right) \Lambda^{-[n]} d \nu,
$$

which is equivalent to

$$
\int f_{2} g_{1} E_{m}\left(g_{2} f_{1}\right) d \nu=\int g_{2} f_{1} H^{\beta[m]} \Lambda^{-[n]} E_{m}\left(f_{2} g_{1} H^{-\beta[m]} \Lambda^{[n]}\right) d \nu, \quad(* * * *)
$$

and this shows lemma 2 for the case $n \geq m$.

Suppose now that $n \leq m$.

From the KMS condition

$$
\begin{gathered}
\phi\left(M_{f_{2}} S^{m}\left(S^{*}\right)^{m} M_{g_{2}} \cdot M_{f_{1}} S^{n}\left(S^{*}\right)^{n} M_{g_{1}}\right)= \\
\phi\left(M_{f_{1}} S^{n}\left(S^{*}\right)^{n} M_{g_{1}} \cdot M_{f_{2}} H^{-\beta[m]} S^{m}\left(S^{*}\right)^{m} H^{\beta[m]} M_{g_{2}}\right) .
\end{gathered}
$$


We point out that in the case $n \leq m$, if $e^{n}=S^{n} S^{* n}$, then $e_{n} e_{m}=$ $e_{m}=e_{m} e_{n}$ and $e^{n} f e^{n}=E_{n}(f) e_{n}$, because $S^{n} S^{* n} f S^{n} S^{* n}=S^{n} \mathcal{L}_{p}^{n}(f) S^{* n}=$ $\alpha^{n}\left(\mathcal{L}_{p}^{n}\right)(f) e_{n}$.

Therefore, from the above and lemma 1 we get for right hand-side of the KMS condition above

$$
\begin{gathered}
\phi\left(M_{f_{1}} S^{n}\left(S^{*}\right)^{n} M_{g_{1}} \cdot M_{f_{2}} H^{-\beta[m]} S^{m}\left(S^{*}\right)^{m} H^{\beta[m]} M_{g_{2}}\right)= \\
\phi\left(M_{f_{1}} e_{n} M_{g_{1}} \cdot M_{f_{2}} H^{-\beta[m]}\left(e_{n} e_{m}\right) H^{\beta[m]} M_{g_{2}}\right)= \\
\phi\left(M_{f_{1}} E_{n}\left(g_{1} \cdot f_{2} H^{-\beta[m]}\right) e_{m} H^{\beta[m]} M_{g_{2}}\right)= \\
\int f_{1} E_{n}\left(g_{1} \cdot f_{2} H^{-\beta[m]}\right) \Lambda^{-[m)} H^{\beta[m]} g_{2} d \nu .
\end{gathered}
$$

Note that for $m \geq n$, we have for any $g_{1}, f_{2} \in C(X)$

$$
\begin{gathered}
E_{n}\left(g_{1} \cdot f_{2} H^{-\beta[m]}\right) H^{\beta[m]}= \\
E_{n}\left(g_{1} \cdot f_{2} H^{-\beta[n]}\left(H^{-\beta[m-n]} \circ T^{n}\right)\right) H^{\beta[n]}\left(H^{\beta[m-n]} \circ T^{n}\right)= \\
E_{n}\left(g_{1} \cdot f_{2} H^{-\beta[n]}\right)\left(H^{-\beta[m-n]} \circ T^{n}\right) H^{\beta[n]}\left(H^{\beta[m-n]} \circ T^{n}\right)= \\
E_{n}\left(g_{1} \cdot f_{2} H^{-\beta[n]}\right) H^{\beta[n]},
\end{gathered}
$$

because $H^{-\beta[m-n]} \circ T^{n} \in \mathcal{R}_{n}$.

Therefore,

$$
\begin{gathered}
\phi\left(M_{f_{1}} S^{n}\left(S^{*}\right)^{n} M_{g_{1}} \cdot M_{f_{2}} H^{-\beta[m]} S^{m}\left(S^{*}\right)^{m} H^{\beta[m]} M_{g_{2}}\right)= \\
\int f_{1} E_{n}\left(g_{1} \cdot f_{2} H^{-\beta[n]}\right) \Lambda^{-[m]} H^{\beta[n]} g_{2} d \nu .
\end{gathered}
$$

Now we consider the left hand side of main equality.

In the same way as before, using $g\left(S^{*}\right)^{n}=\left(S^{*}\right)^{n} \alpha^{n}(g)$,

for $g=\mathcal{L}_{p}^{n}\left(g_{2} f_{1}\right)$ and lemma 2.1 we get

$$
\begin{gathered}
\phi\left(M_{f_{2}} S^{m}\left(S^{*}\right)^{m} M_{g_{2}} \cdot M_{f_{1}} S^{n}\left(S^{*}\right)^{n} M_{g_{1}}\right)= \\
\phi\left(M_{f_{2}} S^{m}\left(S^{*}\right)^{m-n}\left(S^{*}\right)^{n} M_{g_{2}} \cdot M_{f_{1}} S^{n}\left(S^{*}\right)^{n} M_{g_{1}}\right)= \\
\phi\left(M_{f_{2}} S^{m}\left(S^{*}\right)^{m-n} \mathcal{L}_{p}^{n}\left(g_{2} f_{1}\right)\left(S^{*}\right)^{n} M_{g_{1}}\right)= \\
\phi\left(M_{f_{2}} S^{m}\left(S^{*}\right)^{m-n}\left(S^{*}\right)^{n} \alpha^{n}\left(\mathcal{L}_{p}^{n}\right)\left(g_{2} f_{1}\right) M_{g_{1}}\right)= \\
\phi\left(M_{f_{2}} S^{m}\left(S^{*}\right)^{m} \alpha^{n}\left(\mathcal{L}_{p}^{n}\right)\left(g_{2} f_{1}\right) M_{g_{1}}\right)= \\
\int f_{2} g_{1} E_{n}\left(g_{2} f_{1}\right) e_{m} d \nu \\
\int f_{2} g_{1} E_{n}\left(g_{2} f_{1}\right) \Lambda^{-[m]} d \nu .
\end{gathered}
$$


Therefore, the KMS condition implies in the case $n \leq m$

$$
\int f_{2} g_{1} E_{n}\left(g_{2} f_{1}\right) \Lambda^{-[m]} d \nu=\int f_{1} E_{n}\left(g_{1} \cdot f_{2} H^{-\beta[n]}\right) \Lambda^{-[m]} H^{\beta[n]} g_{2} d \nu .
$$

This shows that the KMS condition implies the equality

$$
\int f_{2} g_{1} E_{n}\left(g_{2} f_{1}\right) d \nu=\int g_{2} f_{1} H^{\beta[n]} \Lambda^{-[m]} E_{n}\left(f_{2} g_{1} H^{-\beta[n]} \Lambda^{[m]}\right) d \nu .
$$

Reversing the argument one can show the implication in the other direction.

Therefore, we get equivalence of both conditions and lemma 2.2 is proved.

The following Lemma gives a more simple condition for the $\nu$ defined above by the KMS condition for $\phi$ :

Lemma 2.3: The measure $\nu$ defined as above by the KMS state $\phi$ is characterized by

$$
\int f d \nu=\int E_{m}\left(f \lambda_{m}^{-1}\right) \lambda_{m} d \nu
$$

for any $m \in \mathbf{N}, f \in C(X)$, where $\lambda_{m}=\frac{H^{\beta[m]}}{\Lambda^{[m]}}$.

Proof: The lemma follows from the expression

$$
\int b E_{m}(a) \lambda_{m} d \nu=\int E_{m}(b) a \lambda_{m} d \nu, \quad(* *)
$$

for any $a, b \in C(X)$.

We just have to consider $a=1$ and $b=\frac{f}{\lambda_{m}}$ in the above expression in order to obtain one from the other.

Expression (**) follows from taking (****) with $g_{2}=1, f_{1}=a, g_{1}=$ $1, f_{2}=b \lambda_{m}$

Now we show the existence of $\nu$, and then later we will show by uniqueness that $\nu$ has to be $\nu_{\beta}$.

Remark 3: Note that

$$
E_{m}\left(f \lambda_{m}^{-1}\right)(x)=\alpha^{n}\left(\mathcal{L}_{H, \beta}^{m}(f)\right)(x) .
$$

We will show that there exist a probability $\nu$ such that for any $m$

$$
\int f d \nu=\int E_{m}\left(f \lambda_{m}^{-1}\right) \lambda_{m} d \nu
$$


Proposition 2.1: Consider a fixed $m \in \mathbf{N}$. Suppose $\nu$ is such that for any continuous $f$

$$
\int f d \nu=\int E_{m+1}\left(f \lambda_{m+1}^{-1}\right) \lambda_{m+1} d \nu
$$

then, $\nu$ also satisfies the condition: for any continuous $f$

$$
\int f d \nu=\int E_{m}\left(f \lambda_{m}^{-1}\right) \lambda_{m} d \nu
$$

Proof: Consider $f \in C(X)$.

Under the hypothesis condition for $m+1$ we take $g=E_{m}\left(f \lambda_{m}^{-1}\right) \lambda_{m}$, and we get

$$
\begin{gathered}
\int E_{m}\left(g \lambda_{m}^{-1}\right) \lambda_{m} d \nu=\int E_{m+1}\left(\left(E_{m}\left(f \lambda_{m}^{-1}\right) \lambda_{m}\right) \lambda_{m+1}^{-1}\right) \lambda_{m+1} d \nu= \\
\int E_{m+1}\left(g \lambda_{m+1}^{-1}\right) \lambda_{m+1} d \nu=\int g d \nu
\end{gathered}
$$

As $E_{m}$ is a projection operator over $\mathcal{R}_{m}$, then, for any continuous $g$ we have $\left.E_{m}\left(g H\left(T^{m}(x)\right)^{-\beta}\right)\right)=E_{m}(g) H\left(T^{m}(x)\right)^{-\beta}$.

Therefore,

$$
\begin{aligned}
& \int E_{m+1}\left(E_{m}\left(f \lambda_{m}^{-1}\right) \lambda_{m} \lambda_{m+1}^{-1}\right) \lambda_{m+1} d \nu \\
= & \int E_{m+1}\left(E_{m}\left(f \lambda_{m}^{-1}\right) \frac{H\left(T^{m}(x)\right)^{-\beta}}{\Lambda^{-1}(x)}\right) \lambda_{m+1} d \nu \\
= & \int E_{m+1}\left(E_{m}\left(f \lambda_{m}^{-1} \frac{H\left(T^{m}(x)\right)^{-\beta}}{\Lambda^{-1}(x)}\right)\right) \lambda_{m+1} d \nu .
\end{aligned}
$$

As $E_{m+1} \circ E_{m}=E_{m+1}$, then

$$
\begin{aligned}
& \int E_{m+1}\left(E_{m}\left(f \lambda_{m}^{-1}\right) \lambda_{m} \lambda_{m+1}^{-1}\right) \lambda_{m+1} d \nu \\
= & \int E_{m+1}\left(f \lambda_{m}^{-1} \frac{H\left(T^{m}(x)\right)^{-\beta}}{\Lambda^{-1}(x)}\right) \lambda_{m+1} d \nu \\
= & \int E_{m+1}\left(f \lambda_{m+1}^{-1}\right) \lambda_{m+1} d \nu=\int f d \nu,
\end{aligned}
$$

where we use the $m+1$ condition for $\nu$ and $f$ in last equality.

Therefore,

$$
\int f d \nu=\int g d \nu=\int E_{m}\left(f \lambda_{m}^{-1}\right) \lambda_{m} d \nu
$$


The next result is a particular case of the existence of KMS states presented in section 1 .

Proposition 2.2: Given $\phi$ there exist $\nu$ such that:

a) for all $n \in \mathbf{N}$

$$
\phi\left(M_{f} S^{n}\left(S^{*}\right)^{n}\right)=\int \frac{f}{\Lambda^{[n]}} d \nu
$$

and

b) for all $f_{1}, f_{2}, g_{1}, g_{2} \in C(X)$ and all $n, m \in \mathbf{N}$.

$$
\begin{gathered}
\phi\left(M_{f_{1}} S^{n}\left(S^{*}\right)^{n} M_{g_{1}} \cdot \sigma_{\beta i}\left(M_{f_{2}} S^{m}\left(S^{*}\right)^{m} M_{g_{2}}\right)=\right. \\
\phi\left(M_{f_{2}} S^{m}\left(S^{*}\right)^{m} M_{g_{2}} \cdot M_{f_{1}} S^{n}\left(S^{*}\right)^{n} M_{g_{1}}\right) .
\end{gathered}
$$

\section{Proof:}

Consider the compact set $\mathcal{M}_{n}=\{\nu$ is a probability such that for any continuous $\left.f, \int f d \nu=\int E_{m}\left(f \lambda_{m}^{-1}\right) \lambda_{m} d \nu\right\}$.

From the above Proposition, we get $\mathcal{M}_{n+1} \subset \mathcal{M}_{n}$. Therefore, as the set of all probabilities is compact, there exist $\nu \in \cap_{n=0}^{\infty} \mathcal{M}_{n}$, and therefore, by lemma 2.3 we obtain that $\operatorname{such} \nu$ satisfies the KMS condition.

This shows the existence of $\nu$ and the first part of Theorem 2.1.

The conclusion is that any $\phi$ is associated with a certain $\nu$.

Now we will show the uniqueness of the KMS state:

Theorem 2.2: Given any KMS $\phi$, then $\phi=\phi_{\beta}$ where $\phi_{\beta}$ is the KMS state associated to the Gibbs probability $\nu_{\beta}$.

\section{Proof:}

In order to do that we will show that any possible $\nu$ given by Proposition 2.2 is equal to $\nu_{\beta}$.

Remember that $\lambda_{n}=H^{\beta[n]} \Lambda^{-[n]}$.

Take $\nu$ a probability associated to $\phi$, then for each $n$, and $f \in C(X)$ we have

$$
\int f d \nu=\int E_{n}\left(f \lambda_{n}^{-1}\right) \lambda_{n} d \nu=\int \alpha^{n}\left(\mathcal{L}_{\beta}^{n}(f)\right) \lambda_{n} d \nu .(* * *)
$$

We claim that

$$
\lim _{n \rightarrow \infty} \int E_{n}\left(f \lambda_{n}^{-1}\right) \lambda_{n} d \nu=\int f d \nu_{\beta}
$$

and this shows that $\nu=\nu_{\beta}$, and therefore $\phi=\phi_{\beta}$. 
Now we show the claim. Note that

$$
\int f d \nu=\int E_{n}\left(f \lambda_{n}^{-1}\right) \lambda_{n} d \nu=\int \alpha^{n}\left(\mathcal{L}_{\beta}^{n}(f)\right) \lambda_{n} d \nu=\int \alpha^{n}\left(\frac{\mathcal{L}_{\beta}^{n}(f)}{\lambda_{\beta}^{n}}\right) \lambda_{n} \lambda_{\beta}^{n} d \nu,
$$

where $\lambda_{\beta}$ is the eigenvalue associated to $\mathcal{L}_{\beta}$.

Applying the above expression to $f=h_{\beta}$ (we can assume $h_{\beta}$ is such that $\left.\int h_{\beta} d \nu_{\beta}=1\right)$ and using the fact that $\mathcal{L}_{\beta}^{n}\left(h_{\beta}\right)=\lambda_{\beta}^{n} h_{\beta}$ we get

$$
0<d=\int h_{\beta} d \nu=\int \alpha^{n}\left(h_{\beta}\right) \lambda_{n} \lambda_{\beta}^{n} d \nu
$$

As $h_{\beta}$ is continuous and positive, there exists $c>0$ such for all $x \in X$ we have $h_{\beta}(x)>c$.

From this follow that

$$
d=\int \alpha^{n}\left(h_{\beta}\right) \lambda_{n} \lambda_{\beta}^{n} d \nu>c \int \lambda_{\beta}^{n} \lambda_{n} d \nu
$$

Therefore,

$$
\int \lambda_{\beta}^{n} \lambda_{n} d \nu<d / c
$$

Denote $I=\int f d \nu_{\beta}$.

It is known (see [2]) that uniformly in $z \in X$, we have

$$
\lim _{n \rightarrow \infty} \frac{\mathcal{L}_{\beta}^{n}(f)(z)}{\lambda_{\beta}^{n}}=h_{\beta}(z) I=h_{\beta}(z) \int f d \nu_{\beta} .
$$

Therefore, given $\epsilon>0$, we can find $N>0$ such that for all $n>N$ we have for all $z \in X$

$$
\left|\frac{\mathcal{L}_{\beta}^{n}(f)(z)}{\lambda_{\beta}^{n}}-I_{\beta}(z)\right| \leq \epsilon .
$$

Then, for $n>N$

$$
\begin{gathered}
\left|\int \frac{\alpha^{n}\left(\mathcal{L}_{\beta}^{n}(f)\right)}{\lambda_{\beta}^{n}} \lambda_{n} \lambda_{\beta}^{n} d \nu-\int I \alpha^{n}\left(h_{\beta}\right) \lambda_{n} \lambda_{\beta}^{n} d \nu\right| \leq \\
\int\left|\frac{\alpha^{n}\left(\mathcal{L}_{\beta}^{n}(f)\right)}{\lambda_{\beta}^{n}}(y)-I \alpha^{n}\left(h_{\beta}\right)(y)\right| \lambda_{n}(y) \lambda_{\beta}^{n}(y) d \nu= \\
\int\left|\frac{\mathcal{L}_{\beta}^{n}(f)}{\lambda_{\beta}^{n}}\left(T^{n}(y)\right)-I_{\beta}\left(T^{n}(y)\right)\right| \lambda_{n}(y) \lambda_{\beta}^{n}(y) d \nu \leq \frac{\epsilon d}{c}
\end{gathered}
$$


The conclusion from $(* * *)$ is that for any $f \in C(X)$

$$
\lim _{n \rightarrow \infty} I \int \alpha^{n}\left(h_{\beta}\right) \lambda_{n} \lambda_{\beta}^{n} d \nu=\int f d \nu
$$

Consider now $f=1$ and we get

$$
\lim _{n \rightarrow \infty} \int \alpha^{n}\left(h_{\beta}\right) \lambda_{n} \lambda_{\beta}^{n} d \nu=1 .
$$

From this we conclude that $\int f d \nu=I=\int f d \nu_{\beta}$ for all $f \in C(X)$.

This shows the uniqueness and that $\nu=\nu_{\beta}$.

The final conclusion is that any $\operatorname{KMS} \phi$ for $H, \beta$ is equal to the $\phi_{\beta}$ associated to $\nu_{\beta}$.

\section{Phase Transitions}

We consider here an interesting example of a KMS state associated with the reference measure $\mu$ given by the maximal entropy measure for the shift in 2 symbols $\{0,1\}$. In this case $p=1 / 2$ is contant. We will define a special potential $H$ and we will consider specifically the special value $\beta=1$

We refer the reader to [14] [19] [20] [11] [31] [18] for references and results about the topics discussed in this section.

We are going to introduce the Fisher-Fedenhorf model of Statistical Mechanics in the therminology of Bernouili spaces and Thermodynamic Formalism [14].

We define $\Sigma^{+}$to be the shift space $\Sigma^{+}=\Pi_{0}^{\infty}\{0,1\}$ and denote by $T$ : $\Sigma^{+} \rightarrow \Sigma^{+}$the left shift map. We write $z=\left(z_{0} z_{1} \ldots\right)$ for a point in $\Sigma^{+}$ and $\left[w_{0} w_{1} \ldots w_{k}\right]=\left\{z: z_{0}=w_{0}, z_{1}=w_{1}, \ldots z_{k}=w_{k}\right\}$ for a cylinder set of $\Sigma^{+}$.

We denote by $M_{k} \subset \Sigma^{+}$, for $k>1$, the cylinder set $[\underbrace{111 \ldots 11}_{k} 0]$ and by $M_{0}$ the cylinder set [0]. The ordered collection $\left(M_{k}\right)_{k=0}^{\infty}$ is a partition of $\Sigma^{+}$; in other words these sets are disjoint and their union is the whole space (minus the point $(11 \ldots)$ ). Note that $T$ maps $M_{k}$ bijectively onto $M_{k-1}$ for $k \geq 1$, and onto $\Sigma^{+}$for $k=0$.

The point $(1111 \ldots)$ is fixed for $T$. 
For $\gamma>1$ a fixed real constant, we consider the potential $g(x)$ such that $g(111111 \ldots)=0$,

$$
g(x)=a_{k}=-\gamma \log \left(\frac{k+1}{k}\right),
$$

for $x \in M_{k}$, for $k \neq 0$, and

$$
a_{0}=-\log (\zeta(\gamma))
$$

for $x \in M_{0}$, where $\zeta$ is the Riemann zeta function.

By definition,

$$
\zeta(\gamma)=\left(1^{-\gamma}+2^{-\gamma}+\ldots\right)
$$

and so the reason for defining $a_{0}$ in such way is that, if we define $s_{k}=$ $a_{0}+a_{1}+\cdots+a_{k}$, then $\Sigma e^{s_{k}}=1$.

From now on we assume $\gamma>2$, otherwise we have to consider sigma-finite measures and not probabilities in our problem.

The potential $1<\left(\frac{k+1}{k}\right)^{\gamma}=H(x)=e^{-g(x)}$, for $x \in M_{k}$, is not Hölder and in fact is not of summable variation. Note that $H(1111 \ldots)=1$, The pressure $P(-\log H)=P(g)=P(\log p+\log 2-1 \log H)=0$ and one can show that there exist two equilibrium states for such a potential $g$ (in the sense of minimizing measures for the variational problem): a point mass (the Dirac delta $\delta(111 \ldots))$ at $(1111 \ldots)$, and a second measure which we shall denote by $\tilde{\mu}$ (see [14])

The existence of two probabilities $\tilde{\mu}$ and $\delta_{(1111 \ldots)}$ for the variational problem of pressure defines what is called a phase transition in the sense of Statistical Mechanics [14] [20].

We will describe bellow how to define this measure $\tilde{\mu}$.

Consider as in [14] $\mathcal{L}_{g}^{*}$, the dual of the Ruelle-Perron-Frobenius operator $\mathcal{L}_{g}$ associated to $g$, where the action of $\mathcal{L}_{g}$ on continuous functions is given by

$$
\mathcal{L}_{\beta=1}(\phi)(y)=\sum_{T(x)=y} e^{g(x)} \phi(x) .
$$

The function $P(-\beta \log H)=P(\beta g)$ is strictly monotone for $\beta<1$ and constant equal zero for $\beta>1[\mathrm{H}]$.

We claim that there is a unique probability measure $\nu$ on $\Sigma^{+}$which satisfies $\mathcal{L}_{g}^{*} \nu=\nu[\mathrm{FL}][\mathrm{H}]$. To prove this, note first that $\nu$ cannot have any mass at $(11 \ldots)$; it follows that $M_{0}$ has positive mass, and the stipulation that $\nu$ be an eigenmeasure then gives a recurrence relation for the masses of $M_{k}$. Since $T\left(M_{k}\right)=M_{k-1}$ for $k \geq 1$, we have that the masses of the sets 
in this partition are

$$
\nu(k)=\nu\left(M_{k}\right)=e^{s_{k}}=\frac{(k+1)^{-\gamma}}{\zeta(\gamma)}, k \geq 0 ;
$$

in particular,

$$
\nu(0)=\nu\left(M_{0}\right)=e^{s_{0}}=e^{a_{0}}=\frac{1}{\zeta(\gamma)} .
$$

By the same reasoning, $\nu$ is determined on all higher cylinder sets for the partition $\left(M_{k}\right)_{k=0}^{\infty}$. Hence $\nu$ exists and is unique.

The measure $\nu$ defined above is the unique eigenmeasure for $\mathcal{L}_{\beta=1}^{*}$ and denoted by $\nu_{1}$.

The measure defined by the delta-Dirac on $(111 \ldots)$ is invariant but is not a fixed eigenmeasure for $\mathcal{L}_{g}^{*}$.

This measure $\nu_{1}$ defines a KMS state $\phi_{\nu_{1}}$ for such $H, \beta=1$ and $\mathcal{U}(\mu)$.

We conjecture that there is another KMS state $\phi$ different from $\phi_{\nu_{1}}$ but not associated to a measure. Note that such $H$ assumes the value 1 in just one point.

We define $\tilde{h}(x)$ for $x \in M_{t}$ by

$$
\tilde{h}_{t}=\tilde{h}(x)=\nu(t)^{-1} \sum_{i=t}^{\infty} \nu(i) .
$$

The function $\tilde{h}$ satisfies $\mathcal{L}_{g}(\tilde{h})=\tilde{h}$.

The integral $\int \tilde{h}(x) d \nu_{1}(x)$ is finite if and only if $\gamma>2$. One can normalize $\tilde{h}$, multiplying by a constant $u$ to get $h=u \tilde{h}$ with $\int h d \nu_{1}=1$.

This constant is

$$
u=\frac{1}{\sum_{t=1}^{\infty} t \nu(t-1)}=\frac{\zeta(\gamma)}{\sum_{t=1}^{\infty} t^{1-\gamma}}=\frac{\zeta(\gamma)}{\zeta(\gamma-1)} .
$$

The probability $\tilde{\mu}$ has positive entropy and its support is all $\Sigma^{+}$(see [14] or [20] [11]).

Consider now the invariant probability measure $\tilde{\mu}=h \nu_{1}$. It is known that $\tilde{\mu}$ is an equilibrium state for $-\log H$ in the variational sense $(\beta=1)$ [14]. It is easy to see (because $-\log H(11111 .)=.-\log 1=0)$ that the Dirac-delta measure $\delta_{(11111 \ldots)}$ is also an equilibrium state for $-\log H$ in the variational sense $(\beta=1)$.

The probability $\tilde{\mu}$ has positive entropy and its support is all $\Sigma^{+}$(see [14] or [20] [11]). 
We can conclude from the above considerations that not always an equilibrium probability $\rho$ for the pressure is associated to a KMS state $\phi_{\rho}$ whitout the hypothesis of $H$ and $p$ been Holder. In the present example, this happen because $\rho=\delta_{(1111 \ldots)}$ is not an eigenmeasure of the dual of the Ruelle-Perron-Frobenius operator $\mathcal{L}_{\beta}$ but it is an equilibrium measure for $\beta=1$.

In [19] and [20] the lack of differentiability of the Free energy is analyzed and in [20] [11] [31] it is shown that such systems present polynomial decay of correlation. In [18] it is presented a dynamical model with three equilibrium states.

\section{References}

[1] P. Billingsley, Probability and Measure, Wiley, New York, (1968)

[2] R. Bowen, Equilibrium states and the ergodic theory of Anosov diffeomorphisms, Lecture Notes in Mathematics 470, Springer Verlag, (1975)

[3] R. Bowen and C. Series, Markov maps associated with a Fuchsian group, IHES Publ Math, N 50, pp 153-170 (1979)

[4] O. Bratelli and W. Robinson, Operator Algebras and Quantum Statistical mechanics, Springer Verlag, (1994)

[5] R. Exel, Crossed-Products by finite Index Endomorphisms and KMS states, $J$. Funct. Anal. 199, no. 1, pp 153-188 (2003).

[6] R. Exel, A new look at the Crossed-Products of a $C^{*}$-Algebra by endomorphism, Erg. Theo. Dyn. Syst. 23, no. 6, pp 1733-1750 (2003)

[7] R. Exel, KMS states for generalized Gauge actions on Cuntz-Krieger Algebras, Bull. Braz. Math. Soc. 35, n. 1, pp 1-12, (2004)

[8] R. Exel and M. Lacca, Cuntz-Krieger Algebras for infinite matrices, J. reine angew. Math, 512, pp 119-172, (1999)

[9] R. Exel and M. Lacca, Partial Dynamical Systems and the KMS condition, Comm. Math. Phys. 232, no. 2, pp 223-277 (2003).

[10] R. Exel and A. Lopes, $C^{*}$ Algebras, approximately proper equivalence relations and Thermodynamic Formalism, Erg. Theo. and Dyn. Syst., Vol 24, pp 1051-1082 (2004)

[11] A. Fisher and A. Lopes, Exact bounds for the polynomial decay of correlation, $1 / \mathrm{f}$ noise and the central limit theorem for a non-Holder Potential, Nonlinearity, 14, pp 1071-1104 (2001).

[12] B. Felderhof and M. Fisher, four articles, Ann. Phys. 58, pp 176-281 (1970)

[13] G. Castro and A. Lopes, KMS States, Entropy and a Variational Principle for Pressure, preprint UFRGS (2006)

[14] F. Hofbauer, Examples for the non-uniqueness of the equilibrium states, Trans. $A M S, 228$, pp 133-149 (1977)

[15] V. Jones, Index for subfactors, Invent Math, 72, pp 1-25, (1983)

[16] H. Kosaki, Extensions of Jones' Theory on index to arbitrary subfactors, J. Funct Anal, 66, pp 123-140, (1986) 
[17] M. Lacca, Semigroups of *-endomorphisms, Dirichlet Series and phase transitions, J. Funct Anal, 152, pp 330-378, (1998)

[18] A. Lopes, Dynamics of Real Polynomials on the Plane and Triple Point Phase Transition, Mathematical and Computer Modelling, Vol. 13, № 9, pp. 17-32, 1990.

[19] A. Lopes, A First-Order Level-2 Phase Transition in Thermodynamic Formalism, Journal of Statistical Physics, Vol. 60, № 3/4, pp. 395-411, 1990.

[20] A. Lopes, The Zeta Function, Non-Differentiability of Pressure and The Critical Exponent of Transition", Advances in Mathematics, Vol. 101, pp. 133-167, 1993.

[21] A. Lopes, Entropy, Pressure and Large Deviation, Cellular Automata, Dynamical Systems and Neural Networks, E. Goles e S. Martinez (eds.), Kluwer, Massachusets, pp. 79-146, 1994.

[22] D. Olesen and G. K. Pedersen, Some $C^{*}$-dynamical systems with a single KMS state, Math Scand, 42, pp 111- 118, (1978)

[23] W. Parry and M. Pollicott, Zeta functions and the periodic orbit structure of hyperbolic dynamics, Asterisque, Vol 187-188, pp 1-268 (1990)

[24] G. K. Pedersen, $C^{*}$-Algebras and and their automorphism groups, Acad Press, (1979)

[25] J. Renault, AF-equivalence relations and their cocycles, Operator algebras and mathematical physics (Constança, 2001), Theta, Bucharest, pp 365-377, (2003).

[26] J. Renault, KMS states on $C^{*}$-Algebras associated to expansive maps, Proc. Amer. Math. Soc. 134, no. 7, pp 2067-2078, (2006)

[27] D. Ruelle, Statistical Mechanics of a one-dimensional lattice gas, Comm in Math Phys, 9, pp 267-278, (1968)

[28] D. Ruelle, The thermodynamic Formalism for Expanding maps, Comm in Mat Phys, 125, pp 239-262, (1989)

[29] D. Ruelle, Thermodynamic Formalism, Addison Wesley (1978).

[30] Y. Watatani, Index for $C^{*}$-subalgebras, Mem. Am. Math. Soc. 424, pp 1-117 (1990).

[31] L.-S. Young, Recurrence times and rates of mixing, Israel Jour of Math, 110, pp 153-188, (1997) 